\title{
Characterization of Conducting Polyaniline Blends by Resonance Raman Spectroscopy
}

\author{
José E. Pereira da Silva, Márcia L.A. Temperini and Susana I. Córdoba de Torresi* \\ Instituto de Química, Universidade de São Paulo, CP 26077, 05513-970 São Paulo - SP, Brazil
}

\begin{abstract}
A microscopia óptica juntamente com a espectroscopia Raman no modo microscópico foram utilizadas para investigar as possíveis interações entre a polianilina (PANI) e diferentes polímeros isolantes em diversas blendas condutoras. Interações físicas podem ser inferidas através da análise das intensidades relativas das bandas em 574 e $607 \mathrm{~cm}^{-1}$. A condutividade do material e as intensidades relativas destas bandas Raman são também analisadas.
\end{abstract}

Raman and optical microscopy were used to investigate possible interactions between polyaniline (PANI) and different insulating polymers in conducting blends. Resonance Raman and optical micrographs were used to study the physical interaction in materials. Analysis Raman spectra was done investigating the relative intensity of bands at 574 and $607 \mathrm{~cm}^{-1}$. A relationship between Raman bands and conductivity was also proposed.

Keywords: Raman spectroscopy, optical microscopy, conducting blends, polyaniline

\section{Introduction}

Polyaniline (PANI) is an attractive conducting polymer due to its considerable conductivity, easy synthesis route and good thermal stability. The main disadvantage for industrial use is related to its powdered form, resulting in low processability and poor mechanical properties. A way of overcoming this problem is to prepare blends of PANI with insulating polymers to obtain suitable materials for being used in potential applications.

The mixture of two or more polymers to produce a blend or a composite is an efficient industrial strategy for achieving a material with specific properties. The goal of conducting blends is to combine mechanical properties of insulating polymers and electrical conductivity of conducting ones. These blends can be prepared by chemical (solution casting or melting) or electrochemical methods.

Conductive blends of PANI with poly(methyl methacrylate) (PMMA), ${ }^{1,2}$ polystyrene (PS), ${ }^{3,4}$ polyethylene $(\mathrm{PE})^{5,6}$ and terpolymer ethylene-propylene-diene (EPDM) ${ }^{7}$ were obtained and a great number of practical applications proposed. ${ }^{8-10}$

Optical and electron microscopies are the most widely used techniques for the examination of polymeric blends morphology, but with the intrinsic limitation of providing

\footnotetext{
* e-mail: storresi@iq.usp.br
}

little information about the chemical composition of the phases. On the other hand, Raman spectroscopy used on the microscopic mode is an efficient tool for the characterization of interactions between polymers in the blends. ${ }^{11,12}$ The main advantage of this technique is to achieve information about chemical structure of the different domains in the sample due to high spatial resolution of the instruments $(\sim 1 \mu \mathrm{m})$. Furthermore, it is also possible to obtain information about homogeneity without any previous preparation of the sample. The aim of this paper is to characterize PANI blends, prepared using different parameters (insulating polymers, solvents, temperature and dopants) by Raman spectroscopy and optical microscopy. Results were analyzed by considering chemical or physical interactions between the conductive material and the insulating matrix.

\section{Experimental}

PANI (Scheme 1) was chemically obtained via the oxidative polymerization of aniline (Scheme 2) by ammonium perssulfate in $1.0 \mathrm{~mol} \mathrm{~L}^{-1} \mathrm{HCl}$ according to the procedure described in the literature. ${ }^{13}$ The polymer obtained was de-doped by placing it in a $\mathrm{NH}_{4} \mathrm{OH}$ solution and, after filtering, it was re-doped by dissolution in a 1 mol L-1 camphorsulfonic acid (HCSA) or $1 \mathrm{~mol} \mathrm{~L}^{-1}$ dodecylbenzene sulfonic acid (HDBSA) solutions.

PANI-CSA/PMMA blends were obtained by mixing 


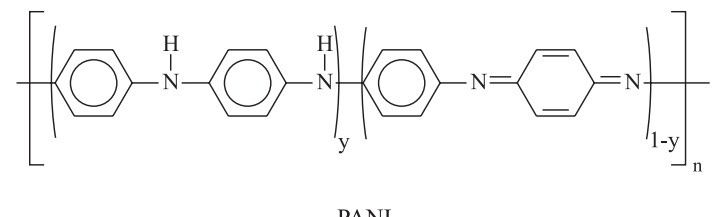

PANI

Scheme 1.<smiles>Nc1ccccc1</smiles>

aniline

Scheme 2.

PANI-CSA and PMMA (Sheme 3) using $m$-cresol (Scheme 4) or $\mathrm{CHCl}_{3}$ as solvents. Solutions of 1, 5, 10 and 20\% of PANI-CSA in PMMA were prepared. PANI-CSA/PS blends were obtained in the same way of PANI-CSA/PMMA ones, but in this case, the dissolution of PS (Scheme 5) is carried out in $m$-cresol heated at $c a .100{ }^{\circ} \mathrm{C}$. After that, the mixture with the conducting polymer was carried out at room temperature.<smiles>CCC(C)(CC)C(=O)OC</smiles><smiles>C=C(C)C(=O)OC</smiles>

poly(methyl methacrylate) methyl methacrylate

Scheme 3.<smiles>Cc1cccc(O)c1</smiles>

$m$-cresol

Scheme 4.<smiles>CCCC(C)c1ccccc1</smiles><smiles>C=Cc1ccccc1</smiles>

polystyrene styrene
PANI-CSA/HDPE (high density polyethylene) blends were obtained by mixing heated solutions of PANI-CSA in $m$-cresol and HDPE (Scheme 6) in decalin $\left(\mathrm{C}_{10} \mathrm{H}_{18}\right)$ (Scheme 7) in the same proportions, according to the literature. ${ }^{5}$ PANI-DBSA/EPDM blends were obtained by mixing conducting and insulating polymers in xylene (Scheme 8) and $\mathrm{CHCl}_{3}$, respectively. In this case, the preparation of the blends involves the heating of EPDM solution in order to dissolve the polymer. The electrical conductivity of the films cast from different solutions was measured by standard four probe method using a Jandel Instrument.

$$
\begin{array}{ll}
\underset{2}{-\mathrm{CH}_{2}-\mathrm{CH}_{2}-\mathrm{Jn}} \quad \mathrm{H}_{2} \mathrm{C}=\mathrm{CH}_{2} \\
\text { polyethylene } & \text { ethylene }
\end{array}
$$

Scheme 6.<smiles>C1CCC2CCCCC2C1</smiles>

decalin

Scheme 7.<smiles>Cc1cccc(C)c1</smiles>

xylene

Scheme 8 .

Raman spectra for $632.8 \mathrm{~nm}$ exciting radiation $(\mathrm{He}-\mathrm{Ne}$ laser, Spectra Physics, mod 127), were recorded on a Renishaw Raman Imaging Microscope (system 3000) containing an Olympus metallurgical microscope and a CCD detector. The laser power at the sample was kept below $\sim 0.7 \mathrm{~mW}$ to avoid thermal degradation. The laser was focused using a 50x objective lens and spatial resolution was about $1 \mu \mathrm{m}$.

\section{Results and Discussion}

Raman spectra of all blends prepared in this work, PANICSA/PMMA, PANI-CSA/PS, PANI-CSA/HDPE, PANI- 
DBSA/EPDM $(20 / 80 \% \mathrm{~m} / \mathrm{m})$ using $\lambda_{\mathrm{o}}=632.8 \mathrm{~nm}$ as the exciting radiation, showed the predominance of bands corresponding to PANI in the doped state while bands related to the insulating polymers can be only observed in Raman spectra of blends containing less than $1 \%$ of conducting polymer. This fact occurs due to the resonance Raman effect, ${ }^{14,15}$ which increases the intensity of the bands of the material when the incident radiation coincides with the frequency of an electronic transition of the sample. In these blends, only PANI absorbs in the visible range; so that, the intensities of PANI doped bands are enhanced compared to those of the insulating polymers.

Figure 1 shows resonance Raman spectra of: (a) pure PANI-CSA, (b) PANI-CSA/PMMA and (c) PANI-CSA/PS blends with $20 \%$ of conducting polymer. Figures 1(d), 1(e) and 1(f) show the enlargement of the same Raman spectra in the wavenumber range marked with a circle. It can be
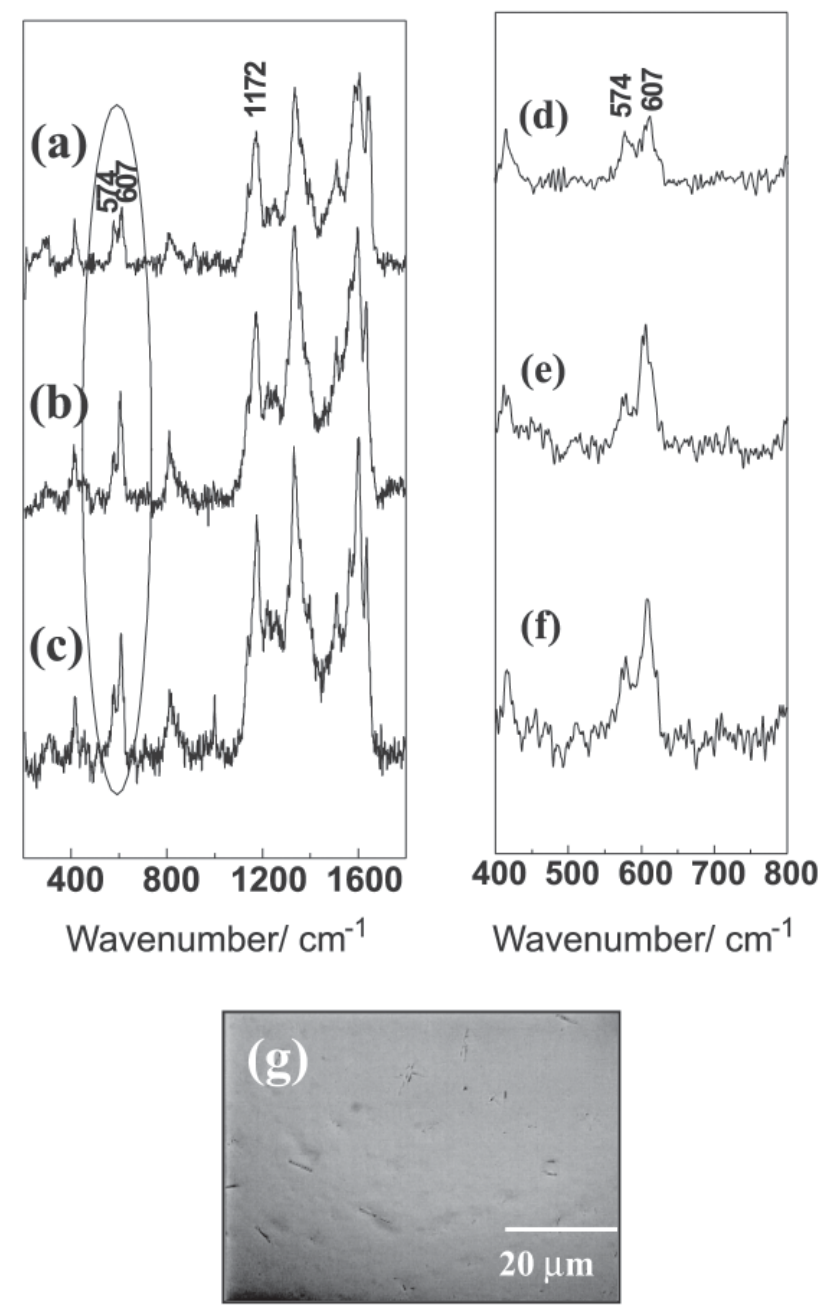

Figure 1. Resonance Raman spectra $\left(\lambda_{0}=632.8 \mathrm{~nm}\right)$ of: (a) pure PANI-CSA, (b) PANI-CSA/PMMA blend $(20 / 80 \% \mathrm{~m} / \mathrm{m}$ ) and (c) PANI-CSA/PS blend $(20 / 80 \% \mathrm{~m} / \mathrm{m})$; (d), (e) e (f) enlargement of the same Raman spectra, but in short range of wavenumber; $(\mathrm{g})$ optical micrograph of PANI-CSA/PMMA blend $(20 / 80 \% \mathrm{~m} / \mathrm{m})$. seen that in the case of pure PANI-CSA $/ m$-cresol, the bands at 574 and $607 \mathrm{~cm}^{-1}$ show the same relative intensities, ${ }^{16}$ while, in the blends, the band at $607 \mathrm{~cm}^{-1}$ is stronger than the $574 \mathrm{~cm}^{-1}$ one. The latter band was assigned to the formation of phenazine like structures produced by crosslinking among PANI-CSA chains (Sheme 9), and it has already seen that this band is strongly enhanced due to resonance phenomena in the red radiation. ${ }^{16}$ It was also shown that changes in some experimental parameters such as temperature, solvents, Fenton's reagents or oxidants in the synthesis of PANI, produce cross-linked PANI chains. ${ }^{16,17}$ The band at $607 \mathrm{~cm}^{-1}$ was assigned to the benzene ring deformation (6a mode $)^{18,19}$ and its increase of intensity has already been observed in in-situ Raman spectra during electrochemical reduction swept. ${ }^{20}$ The decrease of the relative intensity of the band at $574 \mathrm{~cm}^{-1}$ in blends spectra would indicate that a high amount of PMMA or PS within conducting polymer chains avoids the inter-chain interaction of PANI-CSA chains during preparation of the blend; furthermore, these changes are only observed for films that looks homogeneous upon optical microscopy observation, while the behavior for heterogeneous blends is quite different. Figure $1 \mathrm{~g}$ shows the optical micrograph obtained for a PANI-CSA/PMMA $(20 / 80 \% \mathrm{~m} / \mathrm{m})$ film, putting in evidence the high homogeneity of this material. Similar images were obtained for PANI-CSA/PS $(20 / 80 \%$ m/m) blends. Raman spectra of these blends, taken in different regions of the films, do not show relevant changes in the relative intensity of both 607 and $574 \mathrm{~cm}^{-1}$ bands, in good agreement with the homogeneous aspect of the films.

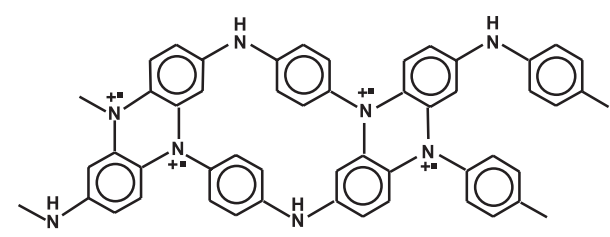

phenazine - like structure

Scheme 9.

Figures $2 \mathrm{a}$ and $2 \mathrm{~b}$ and Figures $3 \mathrm{a}$ and $3 \mathrm{~b}$ show the Raman spectra obtained for PANI-DBSA/EPDM (20/80\% $\mathrm{m} / \mathrm{m}$ ) blends prepared with chloroform or xylene respectively, obtained in different points of the samples. Optical micrographs (Figures $2 \mathrm{c}$ and $3 \mathrm{c}$ ) show the heterogeneous aspect of these materials with agglomerates of conducting polymer in some regions. In all cases, independently on the region where the Raman spectra were taken, it is observed predominately a strong band at 574 $\mathrm{cm}^{-1}$ and a weak shoulder at $607 \mathrm{~cm}^{-1}$. The increase of the 


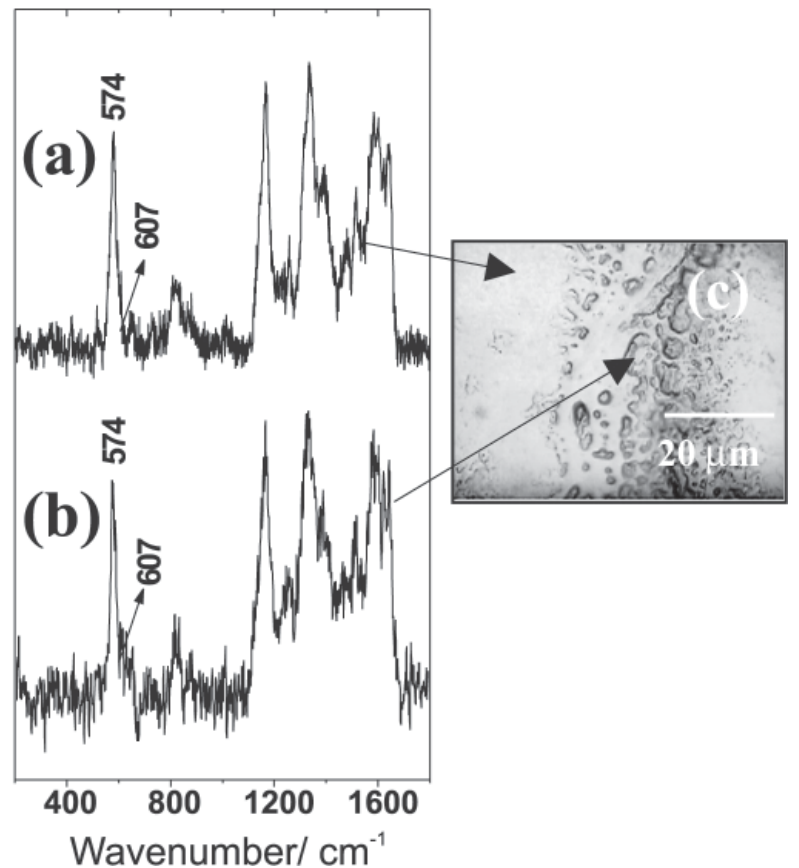

Figure 2. Resonance Raman spectra $\left(\lambda_{0}=632.8 \mathrm{~nm}\right)$ of PANI-DBSA/ EPDM blend $(20 / 80 \% \mathrm{~m} / \mathrm{m})$ prepared in $\mathrm{CHCl}_{3}$ of two different regions: (a) collected in a more homogeneous region and (b) collected in more heterogeneous region; (c) Optical micrograph indicating the acquisitions points.

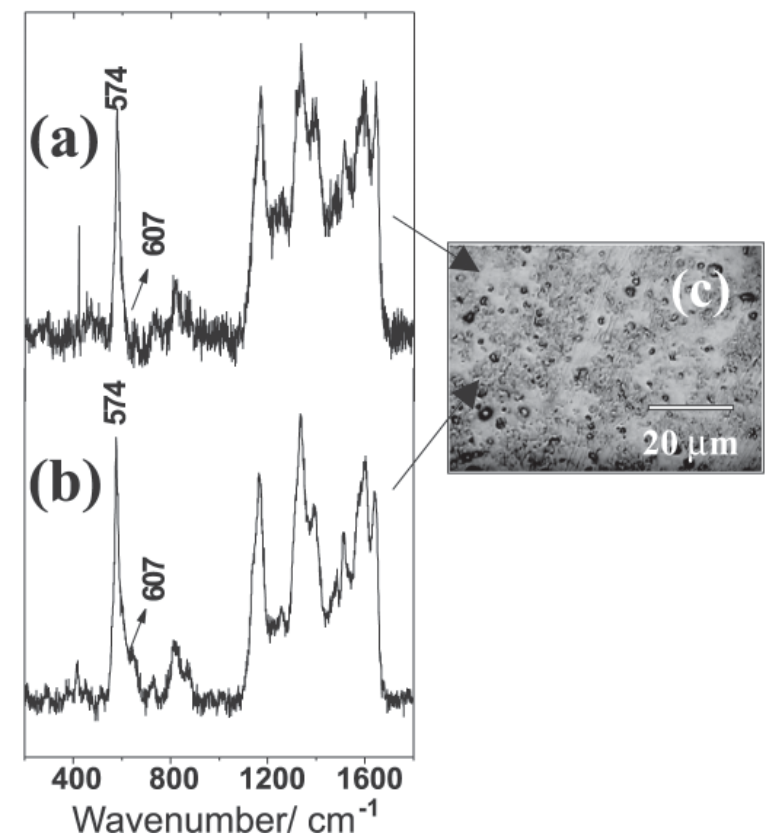

Figure 3. Resonance Raman spectra $\left(\lambda_{0}=632.8 \mathrm{~nm}\right)$ of PANI-DBSA/ EPDM blend $(20 / 80 \% \mathrm{~m} / \mathrm{m})$ prepared in xylene obtained in two different regions: (a) collected in a more homogeneous region and (b) collected in a dark region; (c) Optical micrograph indicating the acquisitions points.

relative intensity of the band at $574 \mathrm{~cm}^{-1}$ indicates a high amount of PANI crosslinked segments due to a higher interchain interaction. ${ }^{16,17}$ Agglomerates shown in the micrographs of PANI-DBSA/EPDM blends are not observed in PANI-CSA/PMMA or PANI-CSA/PS ones, and Raman spectra of these latter materials showed the band at $574 \mathrm{~cm}^{-1}$ weaker than the band at $607 \mathrm{~cm}^{-1}$; thus, it would be possible to do a direct correlation between the relative intensities of these bands and the homogeneity of the different blends studied.

Films of PANI-CSA/HDPE blends were obtained by two methods. In the first case, the preparation was performed by transferring an aliquot of a hot solution blend to a glass substrate by a Pasteur pipette. Raman spectra of this material in different points of the sample (Figures $4 \mathrm{a}$ and $4 \mathrm{~b}$ ) show that the relative intensity of the band at 574 $\mathrm{cm}^{-1}$ is stronger than that at $607 \mathrm{~cm}^{-1}$, indicating the formation of a heterogeneous material, as in the case of PANI-CSA/EPDM blends. The fast solidification of HDPE avoids an intimate mixing between conducting and insulating phases when the hot solution blend was slowly transferred to the Pasteur pipette which was maintained at room temperature. In this way, the penetration of PANI in the insulating matrix is hindered. In agreement with Raman results, the optical micrograph (Figure 4c) shows a heterogeneous material with well localized regions with higher amount of PANI (black points) or higher amount of HDPE (white points).

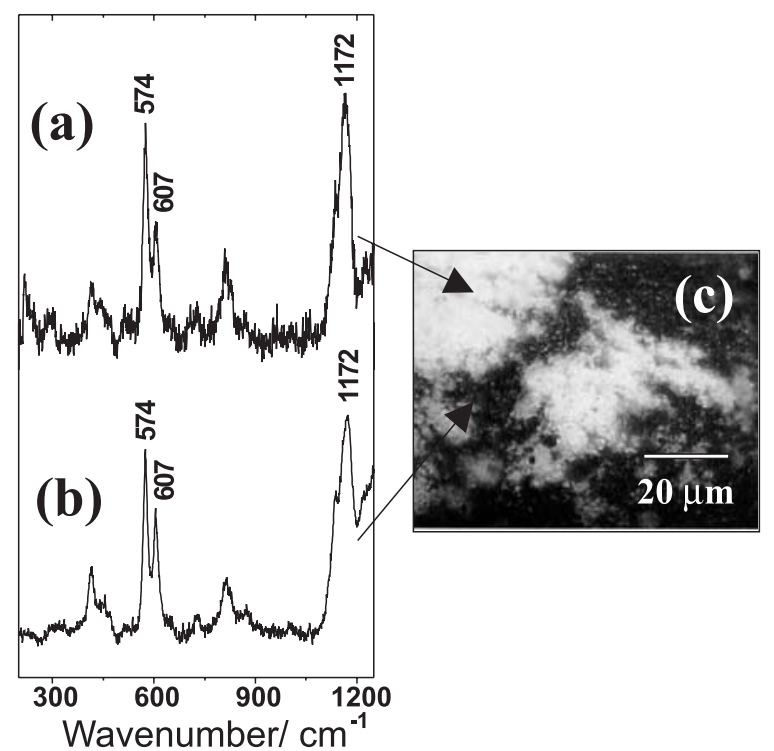

Figure 4. Resonance Raman spectra $\left(\lambda_{0}=632.8 \mathrm{~nm}\right)$ of PANI-CSA/ HDPE $(20 / 80 \% \mathrm{~m} / \mathrm{m})$ heterogeneous blend: (a) collected in a clear region and (b) collected in a dark region; (c) optical micrograph indicating the acquisitions points.

In Figure 5, the Raman spectra of different regions of PANI-CSA/HDPE blends but in another range of wavenumbers, are presented. In this case it is possible to 


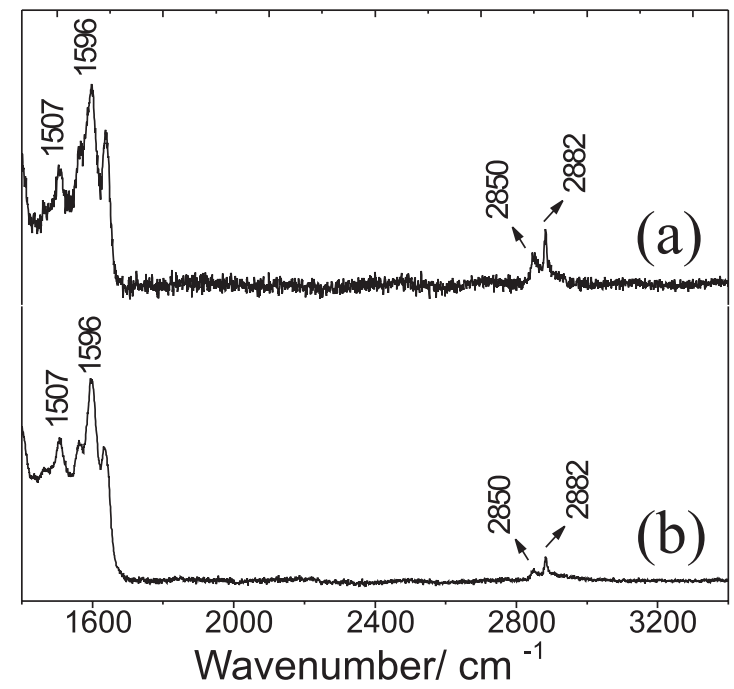

Figure 5. Resonance Raman spectra $\left(\lambda_{0}=632.8 \mathrm{~nm}\right)$ of PANI-CSA/ HDPE $(20 / 80 \% \mathrm{~m} / \mathrm{m})$ heterogeneous blend showing different range of wavenumbers: (a) collected in a clear region; (b) collected in a dark region.

discuss the different compositions of the blend in different points of the sample. Figure 5a shows the Raman spectrum of the blend taken in the white points regions of the material; while, Figure 5b shows the Raman spectrum of the blend taken at black points. First of all, it is interesting to discuss the relative intensities of the bands of HDPE compared with PANI bands. Due to the already discussed Resonance Raman effect that enhances conducting polymer response, it is not possible to observe HDPE bands in the spectral region of characteristic PANI bands (200$1800 \mathrm{~cm}^{-1}$ ). However, the Raman spectra analysis of the blend PANI-CSA/HDPE $(20 / 80 \% \mathrm{~m} / \mathrm{m})$ in another wavenumber range, allows discussing some interesting points due to the presence of polyethylene $\mathrm{C}-\mathrm{H}$ stretching bands in 2850 and $2882 \mathrm{~cm}^{-1}$. By comparing Figures 5a and $5 \mathrm{~b}$, it can be seen that the relative intensities of these bands related to those of PANI located at 1507 and 1596 $\mathrm{cm}^{-1}$ and attributed to the N-H bending and C-C stretching benzene rings, ${ }^{18,19}$ are very different depending on the region of the blend; the relative intensity of HDPE bands being higher in the white points of the sample. On the other hand, black points show a lower relative intensity of HDPE bands and a better signal/noise relationship of the spectrum in agreement with higher amount of PANI in these regions that influences the intensification effect of the signal.

Different results were obtained when PANI-CSA/HDPE blends were prepared by fast transferring the hot mixture to a glass substrate. The Raman spectrum of the material (Figure 6a) shows the intensity of the band at $607 \mathrm{~cm}^{-1}$ being stronger than that of the $574 \mathrm{~cm}^{-1}$, indicating the formation of a homogeneous material of PANI-CSA and HDPE. This fact can be corroborated in the optical micrograph of this blend that does not show phase separation (Figure 6b).
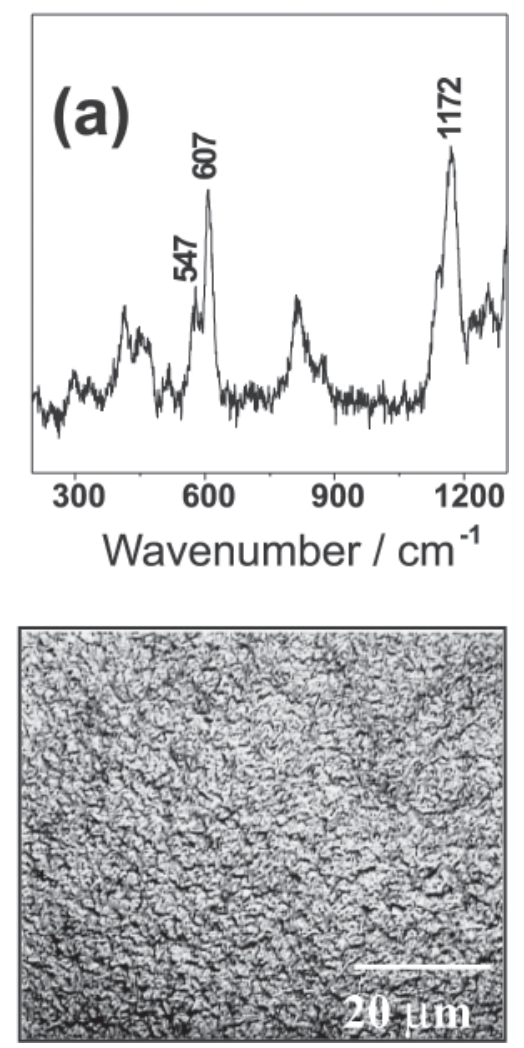

Figure 6. (a) Resonance Raman spectra $\left(\lambda_{0}=632.8 \mathrm{~nm}\right)$ of PANICSA/HDPE $(20 / 80 \% \mathrm{~m} / \mathrm{m})$ homogeneous blend and (b) optical micrograph.

It was also observed a direct relationship between the homogeneity and conductivity of the materials, the conductivity being higher in homogenous than heterogeneous blends with the same amount of PANI-CSA in the insulating matrix Values closed to $10^{-1} \mathrm{~S} \mathrm{~cm}^{-1}$ were obtained for homogeneous PANI-CSA/PMMA and PANICSA/PS $(20 / 80 \% \mathrm{~m} / \mathrm{m})$ blends; on the other hand, the conductivity observed for heterogeneous PANI-CSA/ EPDM $(20 / 80 \% \mathrm{~m} / \mathrm{m})$ blends were of the order of $10^{-5} \mathrm{~S}$ $\mathrm{cm}^{-1}$. Moreover, the value of conductivity obtained for homogeneous PANI-CSA/HDPE was $10^{-1} \mathrm{~S} \mathrm{~cm}^{-1}$; while, PANI-CSA/HDPE heterogeneous blends show low conductivity $\left(10^{-3} \mathrm{~S} \mathrm{~cm}^{-1}\right)$ as observed for heterogeneous PANI-DBSA/EPDM blends. It is important to note that the values of conductivity obtained in this work are close to those found in the literature..$^{1,21,22}$ This is a very important point because it would indicate that Raman spectroscopy would be used as a tool for indicating the conductivity of the material. Figure 7 shows a bar graph depicting the 
relationship of band intensities $\mathrm{I}_{607} / \mathrm{I}_{574}$ for different PANI blends prepared in this work and the conductivity values. Typical $\mathrm{I}_{607} / \mathrm{I}_{574}$ values for homogenous blends are higher than 1.5; while, this value decreases to 0.3 for heterogeneous materials. It can be seen that this relationship is also directly related to the conductivity, as already discussed.

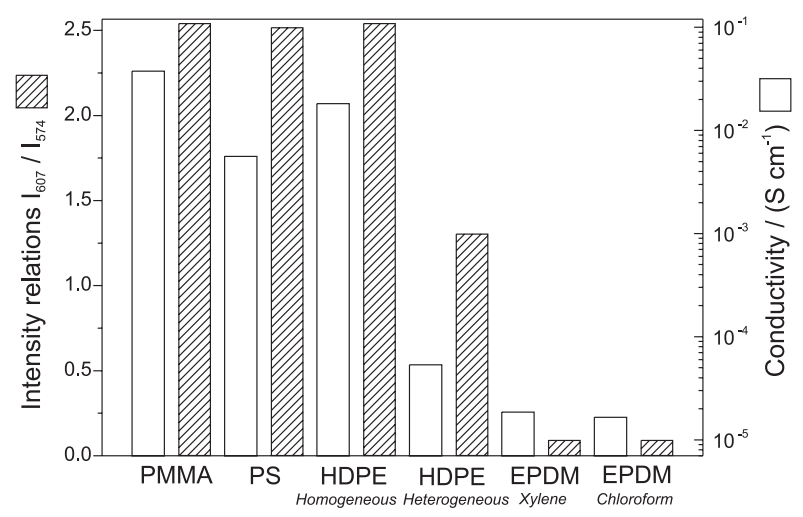

Figure 7. Bar graph of the relationship $\mathrm{I}_{607} / \mathrm{I}_{574}$ intensity bands and conductivity for different blends.

\section{Conclusions}

Raman and optical microscopy were used to investigate possible interactions between PANI and different insulating polymers. Physical interaction was inferred by analyzing the relative intensity of conducting polymer bands at 574 and $607 \mathrm{~cm}^{-1}$ in Raman spectra. The intensity of the band at $574 \mathrm{~cm}^{-1}$ is higher than that at $607 \mathrm{~cm}^{-1}$ when PANI interchain interaction occurs, for example in PANI-DBSA/EPDM blends; while the increase in the intensity of the band at $607 \mathrm{~cm}^{-1}$ indicates a more homogeneous dispersion of conducting material in PMMA, PS and HDPE matrices in agreement with optical micrographs. A relation between Raman bands and conductivity was also proposed. In more homogeneous blends, linked with the increase of $607 \mathrm{~cm}^{-1}$ band, the conductivities values were higher than heterogeneous ones, that are characterized with the increase of $574 \mathrm{~cm}^{-1}$ band.

\section{Acknowledgements}

Authors are indebted to Brazilian Agencies FAPESP (Proc. No 98/15686-3), CNPq and CAPES.

\section{References}

1. Cao, Y.; Smith, P.; MacDiarmid, A. G.; Synth. Met. 1992, 48, 91.

2. Yang, C. Y.; Cao, Y.; Smith, P.; Heeger, A. J.; Synth. Met. 1993, $53,293$.

3. Jousseaume, V.; Morsli, M.; Bonnet, A.; Tesson, O.; Lefrant, S.; J. Appl. Polym. Sci. 1998; 67,1205.

4. Roichman, Y.; Titelman, G.I.; Silverstein, M.S.; Siegman, A.; Markis, M.; Synth Met. 1999, 98, 201.

5. Valenciano, G. R.; Job, A. E.; Mattoso, L. H. C.; Polymer 2000, 41, 4757.

6. Yang, C. Y.; Heeger, A. J.; Cao, Y.; Polymer 2000, 41, 4113.

7. Faez, R.; Gazzoti, W. A.; De Paoli. M. -A.; Polymer 1999, 40, 5497.

8. Wessling, B.; Synth. Met. 1998, 93, 143.

9. Anand, J.; Palaniappan, S.; Sathyanarayana, D. N.; Prog. Polym. Sci. 1998, 23, 993.

10. Roth, S.; Graupner, W.; Synth. Met. 1993, 57, 3623.

11. Tselios, C.; Bikiaris, D.; Maslis, V.; Panayiotou, C.; Polymer 1998, 39, 6807.

12. Ward, Y.; Mi, Y.; Polymer 1999, 40, 2465.

13. Chiang, J. C.; MacDiarmid, A. G.; Synth. Met. 1986, 13, 193.

14. Harris, D. C.; Bertolucci, M. D.; Symmetry and Spectroscopy, Dover Publications, INC: New York, 1989.

15. Sala, O.; Fundamentos da Espectroscopia Raman e no Infravermelho, Ed. Unesp: São Paulo, 1998.

16. Pereira da Silva, J. E.; de Faria, D. L. A.; de Torresi, S. I. C.; Temperini, M. L. A.; Macromolecules 2000, 33, 3077.

17. Rodrigue, D.; Snauwaert P.; Demaret, X; Riga, J.; Verbist, J. J.; Synth. Met. 1991, 41, 769.

18. Louarn, G.; Lapkowski, M.; Quillard, S.; Pron, A.; Buisson, J. P.; Lefrant, S.; J. Phys. Chem. 1996, 100, 6998.

19. Cochet, M.; Louarn, G.; Quillard, S.; Boyer, M. I.; Buisson, J. P.; Lefrant, S.; J. Raman Spectros. 2000, 31, 1029.

20. Pereira da Silva, J. E.; de Torresi, S. I. C.; Temperini, M. L. A.; J. Braz. Chem. Soc. 2000, 11, 91.

21. Valenciano, G. R.; Job, A. E.; Mattoso, L. H. C.; Polymer 2000, 41, 4757.

22. Faez, R.; De Paoli, M. -A.; Eur. Polym. J. 2001, 37, 1139.

Received: November 23, 2004 Published on the web: April 6, 2005

FAPESP helped in meeting the publication costs of this article. 